\title{
Ex Ante Inequality and Under-Nutrition Vulnerability Dynamics: Case Study of the Sundarbans Delta Region, West Bengal, India
}

\author{
Moumita Mukherjee \\ Independent Research Consultant, Atlanta, USA \\ Email: mukherjee.moumita3@gmail.com
}

Received 27 August 2014; revised 30 September 2014; accepted 14 October 2014

Copyright (C) 2014 by author and Scientific Research Publishing Inc.

This work is licensed under the Creative Commons Attribution International License (CC BY).

http://creativecommons.org/licenses/by/4.0/

(c) (i) Open Access

\section{Abstract}

In this paper ex ante inequality measure is used to estimate inequality in childhood chronic under-nutrition among different vulnerable subgroups. Ex ante inequality in nutritional achievement is determined by estimating Concentration Index by ranking the sample population as per different contextual absolute and predicted vulnerabilities. Such vulnerabilities include climatic shock induced asset loss, livelihood insecurity, physical accessibility and consumption sacrifice after treatment seeking of children for under-nutrition related morbidities and perception of care givers regarding quality and effectiveness of care provided by unqualified providers. Results found that vulnerability to consumption poverty aggravated chronic under-nutrition among less vulnerable groups mainly among those who perceived that unqualified providers provided quality service and were very effective during crisis. Whereas, vulnerability to investment poverty due to asset loss aggravates chronic under-nutrition among more vulnerable groups as their low economic resilience against any safety net results in no treatment seeking but rely on home remedies to cure the child. Though due to good social cohesion, traditional knowledge and beliefs for treatment are shared among each other but this is not sufficient to break the under-nutrition morbidity vicious circle, especially when the under-nutrition is chronic in nature. So the paper finally suggests several policy suggestions for different vulnerable segments.

\section{Keywords}

Vulnerability, Concentration Index 


\section{Introduction}

Focus of international development agenda has now been shifted from poor population to vulnerable population in order to reduce the cost of poverty eradication by taking proactive risk management [1]. On the other hand, inequity in several development indicators retards economic growth and holds back reduction in multidimensional poverty. Inequity related policies are trying to control the scenario to sustain economic growth. But inequality in development indicators among vulnerable community may help to reduce such costs further as policies then can protect population subgroups by estimating ex ante inequality in different dimensions of well being. It is still unexplored and therefore it remains the crucial knowledge gap.

Concentration index is a well known tool to estimate socioeconomic inequality in health and healthcare [2]. It helps policy decisions by quantifying the degree of inequity in health status or healthcare among poor and rich. In other words, it estimates how much the worse health or poor healthcare uptake is concentrated among a particular economic group. But in developing countries like India, not only inequity between poor and rich population subgroup is vital; we need to give special attention to the worse pockets of developing states and inequality inside those communities. As for example, though the state of West Bengal in India is a middle level achiever with respect to Human Development indicators [3], several pockets like the Sundarbans Delta Region that epitomizes abject poverty, frequent attack of extreme climatic events, livelihood insecurity presents a painful human face surrounded by natural beauty. As per the monthly consumption expenditure of a typical household in the area, only one third spends Rs. 600 to Rs. 700 with 4 - 5 members and others spend much less than that. Other households spend much less than that amount implying most of the inhabitants spend far less than $\$ 1 /$ day per capita and therefore live below the poverty line. But as they are poor economically (may be categorized as most poor, medium poor and least poor), do we have to assume that there does not exist any inequality within them? Such notion would be wrong.

This paper explores, the degree of inequality in nutritional achievement (of children under the age of five) ranking the sample population by socioeconomic status (SES) first. Then it quantifies the degree of inequality in nutritional status by ranking the sample population according to impact of climate on consumption, SES, livelihood, status of physical accessibility, perception of caregivers regarding quality and effectiveness of healthcare by unqualified providers (as they are the main available providers in the area) and predicted probability of economic status fall. Such alternative ranking is followed to explore how the existing and ex ante inequalities within chronically poor can be captured to identify the different focus areas to design effective policy directives. It will further help to reduce the prevalence of under-nutrition among these particular pockets of concern in order to free the area from under-nourishment and thereby achieve sustainable development.

\section{Review of Literature}

Previous tools on measuring inequality and then equity evolved gradually and serve developing community with new policy directions from time to time. Some of them have major limitations whereas index like Concentration Index has overcome such limitations and are widely used by researchers and policy makers to help sustainability of economic growth [4]. However Concentration Index as defined ranks population or sample population subgroup as per socioeconomic status from poor to rich. What if the population subgroup of interest contains only poor people and they face several contextual risk factors and gives efforts to manage such risks. Therefore such population requires something more for analysis with respect to the relationship between poverty, risk and efforts to manage risk - vulnerability. Several previous research works dealt with such vulnerabilities in isolation of inequalities associated with vulnerable population.

\subsection{Vulnerability Literature}

As discussed in different literature, vulnerability as an ex ante concept is the probability of experiencing loss in the future relative to some benchmark of welfare (may be poverty line) caused by uncertain events like climatic shock, health shock, price shock etc.; the degree of vulnerability depends on the characteristics, frequency and magnitude of the risk and the household's ability to respond to the risk; vulnerability depends on the time horizon; and that the poor and near-poor tend to be vulnerable because of their limited access to assets (broadly defined), higher perceived opportunity cost, weak coping strategies and along with all these if the supply side environment is ineffective or less effective [5]-[10]. 
According to economics literature, the term vulnerability is implicit in the poverty dynamics; majority of economists focus on likelihood of a household's economic status fall below poverty line in future [5] [11]. They estimate income or consumption variance as the measure of vulnerability-outcome of a process of household responses to the risk (ibid.). Difference in income based approach and asset based approach is that in the second one vulnerability is associated with loss or depletion of assets. Such households may not be consumption poor but they are investment poor as their asset base declines over time and are unable or less able to generate surplus to protect, maintain or enhance their assets [12]. The investment poverty concept is forward looking and dynamic [5]. Vulnerability as concept in livelihood literature is defined as the probability that livelihood stress will occur, implying the probability of that household to be deficient in means for mitigating or coping risk without incurring loss after idiosyncratic or covariate shock [13]. Vulnerability in food security literature is defined as the collective effects of "risk and of the ability of an individual or household to cope with those risks and to recover from a shock or deterioration of current status" ([14], p. 9).

But vulnerability is a broader concept encompassing not only any unidimensional volatility, but also includes risks related to health, domestic violence, crime and/or social exclusion [15]. On the other hand, it should take into account the degree of resilience, sensitivity along with susceptibility. Sociologists use the term "vulnerability" to capture the dimensions of poverty which are not captured by money-metric measures. They work on vulnerabilities with respect to capabilities, deprivation or exclusion to determine the process of being poor [16] [17]. In disaster management literature, degrees of vulnerabilities in households, communities etc., include risk and responsiveness to risk [18]. According to their definition of vulnerability “... characteristics of a person or group in terms of their capacity to anticipate, cope with, resist, and recover from the impact of a natural disaster” ([18], p. 9).

Currently, several development literatures deal with how climate change aggravates different natures of vulnerabilities and how to increase the effectiveness of investments in poverty eradication and sustainable development through reduction of such vulnerabilities. Frequent extreme weather event is one feature of climate change that destroys annual harvests, depletes fishing grounds, abolishes forests and make livelihoods insecure and households become highly sensitive and low resilient to such frequent shocks [19]. Economists, climate change specialists and development researchers suggest adaptation strengthening to climate change as the major instrument to build resilience. However, they also suggest that such low resilient regions or communities are in need of social protection or social risk management [1] [20]. As children are more vulnerable compared to other age groups, more focus on children living in such regions are required [21]. As per previous research, it can be done through evidence generation based on which investment on them can be made through social protection as return on such investment is quite high with respect to individual as well as society [21]-[25]. Since, such protection, promotion and prevention will help to break intergenerational transfer of poverty and contribute to economic growth [21] [26].

\subsection{Inequity Literature}

Inequity literature focuses on inequity in health, nutrition and several dimensions of well being by ranking population sub group as per socioeconomic status i.e. it is a development indicator which covers multidimensional aspects of well-being through ex post lens. There are huge research works on inequity in nutritional outcomes which shows poor children are more under-nourished in developing world [27]-[42]. According to these studies, different proximate, underlying and basic factors are responsible for huge gap in nutritional achievement between the rich and the poor where household income at micro level is considered as the underlying factor and poverty is considered as macro level basic factor. In this regard, the Concentration Index is used widely to measure inequity in health, nutritional status and healthcare by ranking the population according to socioeconomic status as developed by the World Bank or with some standardization [2] [4] [43]-[45].

Against this backdrop, it is evident that poor communities face various dimensions of vulnerabilities in different degrees. Till now, capturing the health impact of such vulnerabilities through inequality lens is absent. The present paper is an attempt to quantify it in order to segregate policy interventions for sub-communities in typical vulnerable segments to provide policy suggestions as per need and also to make proactive poverty eradication interventions more cost effective.

Hypothesis of the study: Poor households, who are more vulnerable due to consumption sacrifice; asset loss; increased livelihood insecurity; increased physical inaccessibility after climatic event; and who trust major 
healthcare provider (unqualified providers), perceive poorly about health and nutritional condition of children have more under-nourished children compared to other poor households and also will have higher undernourished children in future.

\section{The Study Area}

The area for the research study was selected based on percentage of poor and marginalized population, geographic location (deltaic or non-deltaic), availability of transport and village infrastructure as it was hypothesized that poor and marginalized people become more vulnerable after climatic shock and shock induced livelihood insecurity, physical inaccessibility and treatment seeking compared to other population subgroup (Figure 1). The Patharpratima block of the Sundarbans Region under the administrative jurisdiction of South 24 Parganas district of West Bengal is the study area. The area consists of 162,300 male population, 154,618 female population, among which 24 percent is Scheduled Caste and 1 percent is Scheduled Tribe population (Projected population from Census 2001) [46]. The selected Gram Panchayets (lower administrative level) from the block-G Plot is the deltaic GP and Ramganga is the non-deltaic GP. In the selected village Rajrajeswarpur of Ramganga GP, 52 percent population and in the another selected village Krishnadaspur of G Plot GP 60 percent population belongs to SC and ST category as per projected population calculated on 2001 Census figure.

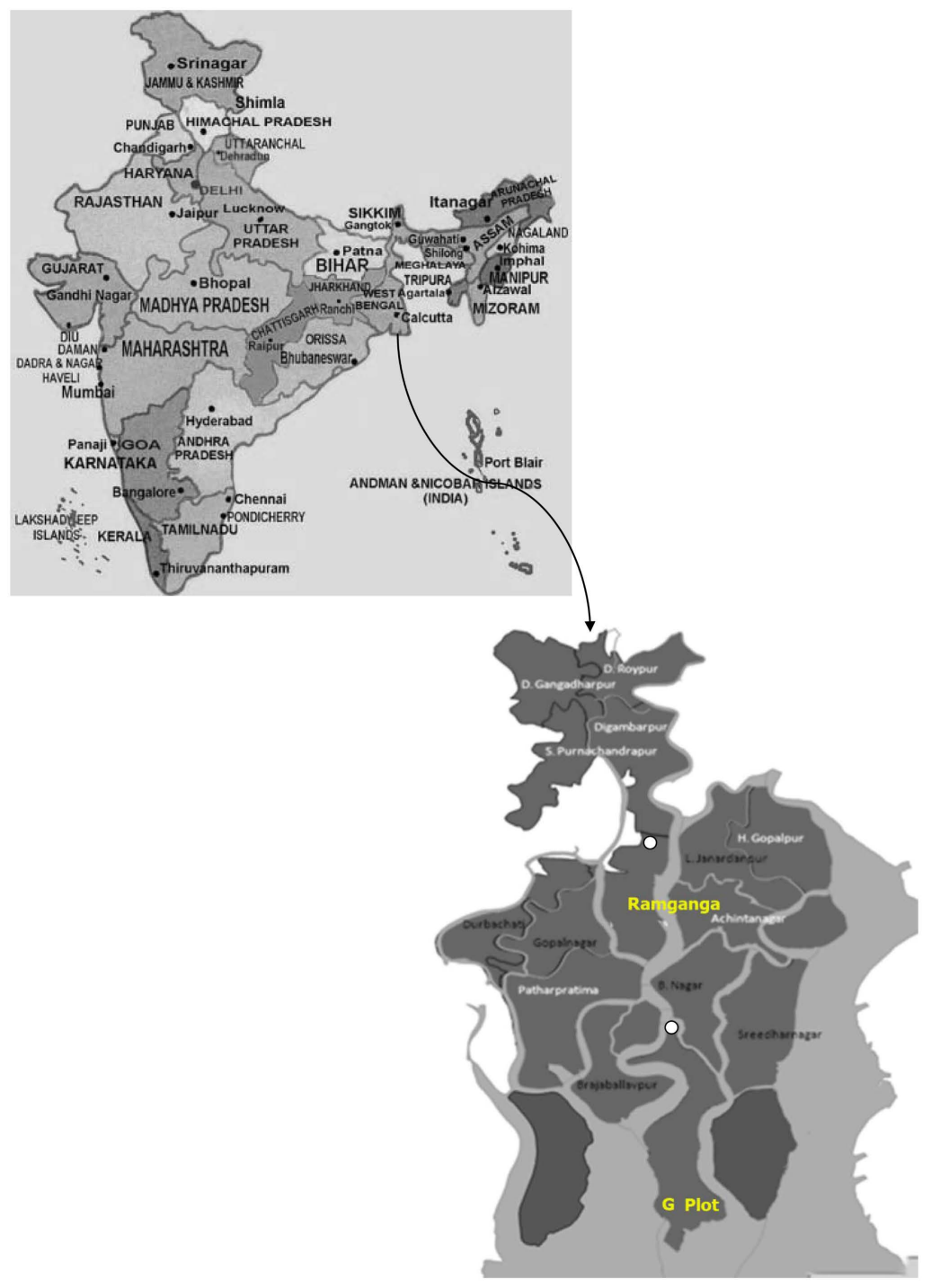

Figure 1. The study area. 
The Sundarbans Delta Region of India is characterized by chronic poverty, insecure livelihood, geographical adversities and extreme climatic events. The geo-volatility of the Sundarbans makes it special not only with respect to livelihood and survival of its people but also in terms of people's health and nutritional status. A typical resident of the Sundarbans carries an extra load of ill-health and health risks compared to others living within the same district. As it has been found, the children are the worst sufferers; most of them are chronically malnourished and, hence, perennially suffer from disproportionately higher burden of respiratory and gastro-enteric troubles [47]. In brief, with about 4 million people currently estimated to live in the region, this neglected population has become a major reservoir for a wide spectrum of health conditions that are not always well recognized by the existing formal health sector (ibid.). In 1973, "Sundarban Development Board" was founded to attend the misery, illiteracy and poor health of the inhabitants, and also for repairing the embankments and transport system. Although quite a few have been implemented, there are still a wide gap between targets and achievements.

In this backdrop, my study is a little initiative to quantify the inequalities in under-nutrition with respect to absolute and estimated contextual vulnerabilities through a pilot study to help the policymakers in designing future enterprise.

\subsection{Data}

To test the study hypothesis, I have collected information on the household level using a structured questionnaire covering socioeconomic and demographic profile of household members; impact of climatic shock on household assets, consumption sacrifice, perception regarding economic status fall, livelihoods, physical infrastructure, and perceived and actual healthcare access; mother and child's health seeking behaviour and nutritional status of children. Also information is collected on what is the amount of healthcare expenditure to cover treatment of their children, whether households' perception about economic status fall differs from actual economic status fall. I have also collected the anthropometric information of children through height and weight measurement.

\subsection{Method}

The data collection is done following stratified random sampling technique where the block is stratified into two strata-deltaic region and non-deltaic region. From deltaic region, I have selected G-Plot gram panchayet which is one of the most vulnerable GPs in the Patharpratima block with respect to geographic location-connection with block headquarter, availability and accessibility of transport. The GP is very much threaten by impacts of climate change induced climatic shock which caused disappearing of one village Gobardhanpur inside the sea 10 - 12 years ago. "The southern part of Sitarampur has disappeared, while the northern part is slowly getting swallowed up as the land gets eroded by the onslaught of the sea.”-The Hindu, Feb 24, 2008. Among 9 villages in G Plot GP, I selected Krishnadaspur based on the criterion of higher marginalized population. Comparatively, Ramganga GP is located in the main land and straight connected to the other developed blocks of the district. So it is comparatively well off with respect to access dimension. Among 10 villages of this GP, I selected Rajrajeswarpur village based on the same criterion.

\section{Sampling}

Observing that deltaic population lives with much geographical adversities compared to non-deltaic population, delta and non-delta stratification under stratified random sampling is justified. After selection of Krishnadaspur and Rajrajeswarpur villages based on the Schedule Caste and Schedule Tribe population and degree of influence of climatic events, I estimated the sample size to test my hypothesis. The sample size calculation is based on three factors i) the child ( 0 - 6 years) population in the block ii) the desired level of confidence and iii) the acceptable margin of error. I collected information for total 338 youngest living children from 338 households. Quantitative data was edited in the field and cleaned. Data analysis was done using Stata 11 package.

Height and weight are measured using the height and weight measuring instruments as recommended by UNICEF.

Analysis is done to see whether contribution to the heterogeneous health and nutrition outcome is attributed to vulnerability related inequality in under-nutrition.

Estimation of inequality after ranking sample households by SES, impact of climatic shock on out of pocket expenditure (OOPE) on healthcare, impact of climatic shock on SES, Livelihood, Physical Accessibility and 
Treatment Seeking Vulnerability due to climatic shock and socio-behavioural factors e.g. perception.

To estimate whether the under-nutrition is more or less likely associated with contextual vulnerabilities, I created Perceived Poverty Index (PPI), Livelihood Insecurity Index (LII), Physical Inaccessibility Index (PII), and Treatment Seeking Perception Index (TSPI). PPI is used as a proxy of SES. PPI is prepared from variables where I asked questions- "In terms of the ability to meet the annual food consumption needs of the household, where would you place your household?", "Have all of your household members including you had full two square meals/sufficient meals everyday for the last seven days?”, "How do you compare your household income-in the last one year in relation to your Household expenditure?” Based on the options, I ran principal component factor analysis which showed scale reliability coefficient is 0.65 and loaded most of the variables in "factor one" and based on this factor score I created Perceived Poverty Index where three categories are most poor, medium poor and least poor.

Impact of climate shock on SES was captured using variables measuring-impact of cyclone and flood on household's house and productive assets. The combined variable is categorized as per perceived poverty trajectory with categories less vulnerable with no or partial damage, vulnerable with partial damage, more vulnerable and devastated. Impact of climatic shock on OOPE is captured through change in healthcare expenditure during climatic shock with categories — not at all, sometimes in devastated family, more often in devastated family, to a great extent in devastated family.

LII was created using the variables measuring the degree of impact (quantifying the quality indicators) of climatic shock on livestock, land and crop, on degree of barrenness of land, difficulty in fishing, livestock rearing, wood cutting or honey collection. The value of scale reliability coefficient is 0.67 . The three categories of first factor score are most vulnerable, medium vulnerable and least vulnerable.

PII is created using variables measuring the degree of impact of climatic shock on village road, transport facility on land and water, access to Government health facility/hospital, caused less/no transport facility or damaged road. Other variables used are inability to access facility due to distance, the mode of transport used to access health facility-walking, by boat, launch, van, small trekker (magic van in local terminology), and bus. The value of scale reliability coefficient is 0.82 . The three categories of first factor score are most difficult to access, medium difficulty to access, least difficulty to access.

TSPI is created using variables quantifying the perception of households regarding availability of medicine and other goods and patient loads in Government hospitals, variation in treatment cost in unqualified provider's clinic during climatic shock, whether health condition of child deteriorates after treatment seeking from unqualified providers, whether keeping children treated under home remedy is better if unqualified provider's treatment is risky as increases frequency of illness, whether treating under qualified provider is better; and the variablespreference pattern about child's delivery when experiencing obstetric complications during climatic shock with options home delivery with dai or unqualified provider or institution, behaviour of unqualified providers during treatment seeking. The scale reliability coefficient is 0.68 . The factor 1 score is categorized into good perception, medium perception and poor perception in relation to choice of provider especially unqualified ones.

The concentration indices are estimated based on ranking the population subgroup from most vulnerable to least vulnerable. In the horizontal axis (x-axis) the cumulative percentage of population ranked by current vulnerabilities or predicted probability of poverty in future and in the y-axis, cumulative percentage of stunting are measured. As the indices of concern are quantifying the inequality in nutritional achievements among children as per their contextual vulnerabilities, therefore the positive value indicates it is disfavouring the least vulnerable and vice versa. Therefore, all the other properties of Concentration Index remaining same, if the vulnerability variable is discrete, where $n$ is sample size, $h$ is the health variable, $\mu$ is mean and $r$ is the fractional rank by vulnerability, then,

$$
\begin{gathered}
C=\frac{2}{n \mu} \sum_{i=1}^{n} h_{i} r_{i}-1-\frac{1}{n} \\
C=\frac{2}{\mu} \operatorname{cov}(h, r)
\end{gathered}
$$

is the Concentration Index where,

$$
C=1-2 \int_{0}^{1} L_{h}(p) d p
$$


$C=1$ if the least vulnerable families have all the stunted children;

$C=-1$ if the most vulnerable families have all the stunted children;

$C=0$ if distribution is proportionate.

\section{Results}

Table 1 shows the Concentration Indices values estimated after ranking the sample by SES (PPI), impact of climate on consumption sacrifice and asset degradation, livelihood (LII), physical inaccessibility (PII) and treatment seeking perception (TSPI). From the above concentration indices, it is visible that, the distribution of nutritional achievement is almost near the equality line implying more or less equal distribution among less and more poor population when I rank the population according to perceived SES (PPI). Therefore, as per CI, ranking the sample by PPI, no significant inequality is visible. CI value, ranking the sample by degree of consumption sacrifice and asset loss after climatic shock shows that stunting is highly concentrated among most vulnerable significantly. So is the results when CI is calculated ranking the sample by LII or PII. When CI is estimated after ranking the sample by caregiver's perception, it is evident that stunting is highly concentrated among families where caregivers perceive that quality and effectiveness of care provided by unqualified provider is "bad" during climatic shock.

Probit model of Table 2 shows that who experienced more consumption sacrifice due to OOPE and asset degradation during climatic shock are significantly more vulnerable to future poverty. This is also true when I controlled impact of climatic shock on SES in the Model 2. When along with it I controlled livelihood insecurity due to climatic shock or impact of climatic shock on physical accessibility to several facilities, vulnerability to poverty is significantly higher among those households who did consumption sacrifice because incurred higher cost on healthcare during climatic shock for child's ailment and also faced asset degradation. When along with all these factors, caregiver's perceived quality and effectiveness of care is controlled, it is evident that families where caregivers possess perception "good" about quality and effectiveness of unqualified provider are more vulnerable to economic status fall.

Moreover, Concentration Indices are also estimated after ranking the sample by predicted vulnerabilities estimated from Probit models-Model 1 to Model 5 respectively (Table 3). After predicting the vulnerability of the household to future economic status fall due to consumption sacrifice after OOPE for child's treatment during or after climatic shock, then ranking the sample by such degree of vulnerabilities, stunting is likely to be more concentrated among least (contextually) vulnerable households and where caregivers' perception regarding quality and effectiveness of care provided by unqualified provider is "good" during climatic shock.

In the multinomial logit model of Table 4, households with no or partial damage to assets has been taken as base outcome. Less or more devastated families are compared with the base and it is observed that families who face most difficulty to access basic facilities during climatic events, have done severe consumption sacrifice for covering treatment of common childhood ailments are more vulnerable to future asset poverty.

In the Table 5, Concentration Indices are also estimated after ranking the sample by predicted vulnerabilities estimated from Multinomial Logit Models-Model 1 to Model 3 respectively. After predicting the vulnerability of the household to future economic status fall due to asset loss after climatic shock, then ranking the sample by such degree of vulnerabilities, stunting is likely to be more concentrated among more (contextually) vulnerable households.

\section{Table 1. Measuring different inequalities in under-nutrition based on different rankings—Concentration Indices.}

\begin{tabular}{cc} 
Ranking of sample by & Concentration Index \\
\hline SES (PPI) & $-0.008^{* * *}$ \\
Impact of climate on consumption sacrifice and asset degradation & $-0.188^{* * *}$ \\
Livelihood Insecurity Index & $-0.124^{* * *}$ \\
Physical Inaccessibility Index & $-0.176^{* * *}$ \\
Treatment Seeking Perception Index & $0.204^{* * *}$ \\
\hline
\end{tabular}

${ }_{\mathrm{p}} \mathrm{p}<0.1 ;{ }^{* *} \mathrm{p}<0.05 ;{ }^{* * *} \mathrm{p}<0.01$. 
Table 2. Multivariate probit model to assess the influence of contextual vulnerabilities on the likelihood of actual economic status fall measured by consumption sacrifice after OOPE on healthcare for children (odds ratios are presented in the table below).

\begin{tabular}{|c|c|c|c|c|c|}
\hline & Model 1 & Model 2 & Model 3 & Model 4 & Model 5 \\
\hline \multicolumn{6}{|c|}{ OOPE increases after climatic event with ref. category-not at all } \\
\hline With no or partial damage & $4.33^{* * *}$ & $3.36^{*}$ & $4.32^{*}$ & $4.18^{*}$ & (Omitted) \\
\hline sometimes in devastated family & $3.80^{* * * *}$ & $4.44^{* * *}$ & $4.51^{* * *}$ & $4.52^{* *}$ & 2.96 \\
\hline More often in devastated family & $4.28^{* * *}$ & $5.49^{* * * *}$ & $5.60^{* * *}$ & $4.64^{* * * *}$ & (Omitted) \\
\hline To a great extent in devastated family & $6.98^{* * *}$ & $7.11^{* * *}$ & $7.24^{* * *}$ & $5.39^{* *}$ & (Omitted) \\
\hline \multicolumn{6}{|c|}{ Asset degradation after climatic event occurs among households with ref category—less vulnerable } \\
\hline More vulnerable with no or partial damage & & 1.21 & 0.91 & 0.55 & 0.79 \\
\hline More vulnerable and devastated & & 0.84 & 1.04 & 0.85 & 0.98 \\
\hline \multicolumn{6}{|c|}{ Ref. category—most vulnerable to livelihood insecurity after climatic shock } \\
\hline Medium insecure livelihood after climatic shock & & & 0.62 & $0.29^{* *}$ & 0.58 \\
\hline Least insecure livelihood after climatic shock & & & 1.15 & 0.37 & 0.38 \\
\hline \multicolumn{6}{|c|}{ Influence of accessibility hamper after climatic shock with ref. category-most difficult to access } \\
\hline Moderate difficult to access & & & & 1.76 & 3.22 \\
\hline Less difficult to access & & & & $4.57^{* * * *}$ & 2.64 \\
\hline \multicolumn{6}{|c|}{$\begin{array}{l}\text { Perception of caregivers regarding quality of care (unqualified provider during or after climatic shock) } \\
\text { with ref. category-poor perception }\end{array}$} \\
\hline Medium perception & & & & & $0.01^{* * *}$ \\
\hline Poor perception & & & & & $0.02^{* * *}$ \\
\hline Number of observations & 313 & 304 & 249 & 240 & 35 \\
\hline Wald chi ${ }^{2}$ & 30.78 & 32.59 & 32.24 & 27.32 & 336.75 \\
\hline Prob $>$ chi $^{2}$ & 0.0000 & 0.0000 & 0.0001 & 0.0023 & 0.0000 \\
\hline pseudo $\mathrm{R}^{2}$ & 0.1593 & 0.1816 & 0.2365 & 0.2922 & 0.4507 \\
\hline
\end{tabular}

${ }^{*} \mathrm{p}<0.1 ;{ }^{* *} \mathrm{p}<0.05 ;{ }^{* * *} \mathrm{p}<0.01$.

Table 3. Measuring different inequalities in under-nutrition based on different predicted vulnerability rankings-Concentration Indices.

Based on estimated vulnerability rank from probit models

Impact of climatic shock on OOPE

Impact of climatic shock on OOPE, controlling the climatic impact on SES

Impact of climatic shock on OOPE, controlling the climatic impact on SES, livelihood

Impact of climatic shock on OOPE, controlling the climatic impact on SES, livelihood, physical accessibility

Impact of climatic shock on OOPE, controlling the climatic impact on SES, livelihood, physical accessibility and perception regarding quality and effectiveness of unqualified providers during climatic shock
Concentration Index

$0.034^{* *}$

$0.040^{* * *}$

$0.008^{* *}$

$-0.029$

$-0.385^{* * *}$

${ }^{*} \mathrm{p}<0.1 ;{ }^{* *} \mathrm{p}<0.05 ;{ }^{* * *} \mathrm{p}<0.01$. 
Table 4. Multinomial logit model to assess the influence of contextual vulnerabilities on the likelihood of actual economic status fall measured by asset loss after climatic shock (relative risk ratios are presented in the table below).

\begin{tabular}{|c|c|c|c|}
\hline & Model 1 & Model 2 & Model 3 \\
\hline \multicolumn{4}{|c|}{ Less devastation vs. no or partial damage } \\
\hline \multicolumn{4}{|c|}{ Ref. category-most vulnerable to livelihood insecurity after climatic shock } \\
\hline Medium insecure livelihood after climatic shock & 1.09 & 2.78 & 2.46 \\
\hline Least insecure livelihood after climatic shock & $0.10^{* * *}$ & 0.40 & 0.45 \\
\hline \multicolumn{4}{|c|}{ Ref. category—influence of accessibility hamper after climatic shock with ref. category—most difficult to access } \\
\hline Moderate difficult to access & & 0.43 & $0.16^{*}$ \\
\hline Less difficult to access & & $0.03^{* * *}$ & $0.01^{* * *}$ \\
\hline \multicolumn{4}{|c|}{ Ref. category-poor with severe consumption sacrifice } \\
\hline Moderate poor with moderate consumption sacrifice & & & 0.33 \\
\hline Less poor with moderate consumption sacrifice & & & $0.10^{* *}$ \\
\hline \multicolumn{4}{|c|}{ Mostly devastated vs. no or partial damage } \\
\hline \multicolumn{4}{|c|}{ Ref. category-most vulnerable to livelihood insecurity after climatic shock } \\
\hline Medium insecure livelihood after climatic shock & 1.55 & 2.12 & 2.13 \\
\hline Least insecure livelihood after climatic shock & 0.53 & 0.84 & 0.78 \\
\hline \multicolumn{4}{|c|}{ Ref. category—influence of accessibility hamper after climatic shock with ref. category—most difficult to access } \\
\hline Moderate difficult to access & & 0.42 & $0.18^{*}$ \\
\hline Less difficult to access & & 0.33 & $0.09^{* *}$ \\
\hline \multicolumn{4}{|c|}{ Ref. category—poor with severe consumption sacrifice } \\
\hline Moderate poor with moderate consumption sacrifice & & & 0.65 \\
\hline Less poor with moderate consumption sacrifice & & & $0.15^{* *}$ \\
\hline Number of observations & 267 & 258 & 233 \\
\hline Wald chi ${ }^{2}$ & 17.99 & 33.21 & 41.28 \\
\hline Prob $>$ chi $^{2}$ & 0.0012 & 0.0001 & 0.0000 \\
\hline Pseudo $\mathrm{R}^{2}$ & 0.0497 & 0.1051 & 0.1386 \\
\hline
\end{tabular}

${ }_{\mathrm{p}}^{*}<0.1 ;{ }^{* *} \mathrm{p}<0.05 ;{ }^{* * *} \mathrm{p}<0.01$

Table 5. Measuring different inequalities in under-nutrition based on different predicted vulnerability rankings as per asset degradation-Concentration Indices.

Based on estimated vulnerability due to asset degradation-rank from multinomial logit models

The climatic impact on livelihood

Impact of climatic shock on livelihood, controlling physical accessibility

Impact of climatic shock on livelihood, controlling physical accessibility, consumption sacrifice
Concentration Index

$-0.058$

$-0.132^{* * *}$

$-0.196^{* * *}$ ${ }_{\mathrm{p}}^{*}<0.1 ;{ }^{* *} \mathrm{p}<0.05 ;{ }^{* * *} \mathrm{p}<0.01$.

\section{Discussion}

The present paper analyses vulnerability related inequality in childhood under-nutrition after looking at its influence on future economic status (measured by consumption sacrifice due to treatment of under-nutrition re- 
lated morbidities or asset loss after climatic shock) - therefore the paper's contribution is in the area of undernutrition-vulnerability dynamics. Such linkage will now be discussed with focus on developing countries in general and India in particular to help policy directives for current and future vulnerable communities.

Analyses are done from two perspectives-consumption poverty and investment poverty. According to the vulnerability to consumption poverty, households who are less vulnerable to poverty, likelihood of chronic under-nutrition is higher among their children and children whose caregivers perceive that unqualified medical practitioners are more effective and provide good quality care are likely to be more stunted. Therefore, though likelihood of future status fall describes the inequality in nutritional status, this factor is not sufficient to explain the vulnerability to become under-nourished. Service delivery effectiveness and availability of good quality care is major important factor to reduce vulnerability to be under-nourished as people face out of pocket expenses more relying on unqualified practitioners. According to the investment poverty perspective, likelihood of stunting is higher among more contextually vulnerable subgroup.

Regarding vulnerability to consumption poverty, it is usually visible in other developing countries that vulnerable less-poor families are more prone to transient bouts of poverty after any shock [48] [49]. In our study area, when they face climate shock induced health shocks like diarrhea among their children, they first visit nearby unqualified providers due to local connections, trust and availability of treatment either in credit or in package system as found in earlier studies [47]. But as it is evident in our study, some of them experience worsening of child's health or recurrence of the disease and therefore search for costlier private provider because service of Government facility is sub-optimal in quantity. The study also shows that the cost they incur for treatment doubles and they cope with such situation through consumption sacrifice as safety nets are almost unavailable. Such a situation makes them vulnerable to future poverty as also evident in previous studies in rural Ethiopia or Vietnam [8] [50].

On the other hand, households who face asset loss due to climatic shock and low resilient due to living under extreme poverty, they even cannot go for any treatment options after such health shocks but rely on home remedies to cure the child. Though due to good social cohesion, traditional knowledge and beliefs for treatment are shared among each other but this is not sufficient to break the under-nutrition morbidity vicious circle, especially when the under-nutrition is chronic in nature as visible in supporting qualitative study. Supporting this argument, as found in an in-depth anthropological study on variation in the household management of child diarrhea in 3 villages in rural North India; qualitative and quantitative results showed both positive and negative diarrhea management behaviors where the study used to collect data on a series of variables, including maternal knowledge, beliefs, and practices during diarrhea, feeding and fluid intake during diarrhea, treatment choices, and knowledge and use of oral rehydration therapy [51].

Hence vulnerability due to health shocks remains crucial for developing nations like India in the absence of safety net measures that might have protected the households from being vulnerable to such health shocks; more specifically for the ones those share weak endowments. Side by side, Vulnerability to worse health and nutritional status preceded by asset loss due to big covariate shock e.g. climatic shock or consumption sacrifice due to idiosyncratic health shock or this idiosyncratic consumption shock if is preceded by that large investment shock make households vulnerable to poverty - transient or chronic-as well as such economic vulnerabilities make the child population vulnerable to chronic under-nutrition. Therefore as per vulnerability sub-groups, specific policy focuses are required to shatter the poverty-vulnerability-chronic under nutrition-morbidity vicious circle. Below some context specific policy suggestions are provided.

Policy suggestion for less vulnerable subgroup facing consumption volatility after OOPE on healthcare:

1) Micro credit availability for treatment seeking as per the monthly income-can reduce the risk of consumption reduction.

2) Making available the doctors of alternative medicines in remote village areas as substitutes or in hard to reach areas as complements to unqualified providers will reduce the risk of recurrence morbidity and related OOPE.

3) Institutionalizing unqualified providers through proper monitoring and reporting system can reduce the risk of vulnerability to transient poverty and under-nutrition.

Policy suggestion for more vulnerable subgroup facing asset volatility after climatic shock:

1) Helping to learn the community adaptation strategies from within the community to best cope with the disaster.

2) Introduction of community level social insurance to build contingency and monitoring through community 
based associations.

3) Protection of community resources and repairing and cleaning them time to time with proper monitoring e.g. storing drinking water throughout the year to lower the safe drinking water crisis during climatic events, cleaning the source area, following hygienic behaviour during childcare.

4) Increasing the effectiveness of behaviour change activities within community.

Policy suggestion for more vulnerable subgroup facing asset volatility after climatic shock followed by consumption dispersion due to climatic event aggravated health shock:

1) Following scientific way to reduce salinity in land so that climatic shock cannot affect the harvesting cycle.

2) Protecting livestock in separate strongly built house and diversifying livelihood by encouraging poultry farming, tailoring and weaving with direct interaction with urban market (though community people has already adopted these source of incomes but they get very low price for these products as except some of them, majority cannot go to the urban area for sale to grab the better price).

3) Regulating food and healthcare market through

a) Price ceiling of major food crops and monitoring it effectively.

b) Making the essential medicines easily available.

c) Providing training to unqualified providers and monitoring them on regular basis to minimize the risk.

d) Quality and effectiveness measure of Integrated Child Development Services and healthcare provider in fixed intervaland strengthening of monitoring is required.

4) Creating more involvement of community through active cooperation of SHGs will help in community mobilization.

5) Weekly meeting on contingency plan and procurement, among mothers and grandmothers of young children with health and nutrition workers should be arranged and monitored.

\section{Conclusion}

Application of ex ante inequality in empirical study like the current work will help to build policies to prevent households from slipping into the deeper poverty in future. It also predicts the ex ante concentration of chronic under-nutrition among children to help the policymakers in developing strategy to stop intergenerational transfer of poverty and under-nutrition and help to sustain economic growth over a longer period of time. Therefore such predictions and steps towards prevention are more cost effective than poverty eradication strategies. Further research is required to estimate the amount of costs that can be saved as well as how much quality of life can be saved adjusting the vulnerabilities.

\section{Acknowledgements}

I express my appreciation for the financial support provided by the Sir Ratan Tata Trust to explore the research work. I dedicate my work to my mother project Future Health Systems Research Project Consortium and specially to my Doctoral Supervisor Prof. Barun Kanjilal since only because of them I came to know about the 'human face' of the Sundarbans and started thinking of working on it following them. I also express my gratitude to eminent professors and scholars of Indian Statistical Institute, Kolkata, Prof. Subha Ray, Department of Anthropology, University of Calcutta in enriching my field work tools and methodology.

\section{References}

[1] Holzmann, R. and Jorgensen, S. (1999) Social Protection as Social Risk Management: Conceptual Underpinnings for the Social Protection Sector Strategy Paper. The World Bank, Washington DC.

[2] O’Donnell, O., Van Doorslaer, E., Wagstaff, A. and Lindelow, M. (2008) Analyzing Health Equity Using Household Survey Data. The World Bank, Washington DC.

[3] United Nations Development Programme (2011) India Human Development Report.

[4] Koolman, X. and van Eddy, D. (2004) On the Interpretation of a Concentration Index of Inequality. Working Paper \#4, ECuity II Project.

[5] Alwang, J., Siegel, P.B. and Jorgensen, S.L. (2001) Vulnerability: A View from Different Disciplines. Social Protection Discussion Paper Series. The World Bank, Washington DC.

[6] Ellbers, C. and Gunning, J. (2003) Growth and Risk: Methodology and Micro-Evidence. Tinbergen Institute Discus- 
sion Papers, 03-068/2.

[7] Ligon, E. and Schechter, L. (2003) Measuring Vulnerability. The Economic Journal, 113, C95-C102. http://dx.doi.org/10.1111/1468-0297.00117

[8] Calvo, C. and Dercon, S. (2005) Measuring Individual Vulnerability. University of Oxford. Discussion Paper Series No. 229.

[9] Baeza, C. and Packard, T. (2006) Beyond Survival Protecting Households from Health Shocks in Latin America. The World Bank, Washington DC. http://dx.doi.org/10.1596/978-0-8213-6571-7

[10] Azam, S. and Imai, K.S. (2009) Vulnerability and Poverty in Bangladesh. Chronic Poverty Research Centre.

[11] Ravallion, M. (1998) Poverty Lines in Theory and Practice. Living Standards Measurement Study, Working Paper No. 133, World Bank, Washington DC.

[12] Reardon, T. and Vosti S. (1995) Links between Rural Poverty and the Environment in Developing Countries: Asset Categories and Investment Poverty. World Development, 23, 1495-1506. http://dx.doi.org/10.1016/0305-750X(95)00061-G

[13] Chambers, R. (1989) Editorial Introduction: Vulnerability, Coping and Policy. IDS Bulletin, 20, 1-7. http://dx.doi.org/10.1111/j.1759-5436.1989.mp20002001.x

[14] Maxwell, D., Levin, C., Armar-Klemesu, M., Ruel, M., Morris, S. and Ahaideke, C. (2000) Urban Livelihoods and Food and Nutrition Security in Greater Accra, Ghana. Research Report 112, International Food Policy Research Institute (IFPI), Washington DC.

[15] Coudouel, A. and Hentschel, J. (2000) Poverty Data and Measurement. Preliminary Draft for a Sourcebook on Poverty Reduction Strategies. The World Bank, Washington DC.

[16] Moser, C. and Holland, J. (1997) Household Responses to Poverty and Vulnerability, Volume 4: Confronting Crisis in Cawama, Lusaka, Zambia. Urban Management Programme Policy Paper, UMPP No. 24. The World Bank, Washington DC. http://dx.doi.org/10.1596/0-8213-3850-1

[17] Bebbington, A. (1999) Capitals and Capabilities: A Framework for Analyzing Peasant Viability, Rural Livelihoods and Poverty. World Development, 27, 2021-2034. http://dx.doi.org/10.1016/S0305-750X(99)00104-7

[18] Blackie, P., Cannon, T., Davis, I. and Wisner, B. (1994) At Risk: Natural Hazards, People’s Vulnerability and Disasters. Routledge, New York.

[19] The World Bank (2009) Poverty and Climate Change Reducing the Vulnerability of the Poor through Adaptation. Washington DC.

[20] Mukherjee, M., Kanjilal, B., Barman, D. and Mazumdar, P.G. (2012) Child Health in the Sundarbans: How Far Mutually Reinforcing Shocks Act as Contextual Determinants? Journal of Health Management, 14, 117-140. http://dx.doi.org/10.1177/097206341201400203

[21] Sanfilippo, M., de Neubourg, C. and Martorano, B. (2012) The Impact of Social Protection on Children: A Review of the Literature. Working Paper 2012-06, UNICEF Office of Research, Florence.

[22] Asian Development Bank (2001) Social Protection in Asia and the Pacific. Asian Development Bank, Manila.

[23] Fajth (2009) Joint Statement on Child Sensitive Social Protection. Presentation at Social Protection Floor Initiative, Turin.

[24] UNICEF, et al. (2009) Joint Statement on Advancing Child-Sensitive Social Protection. http://www.unicef.org/aids/files/CSSP_joint_statement_10.16.09.pdf.

[25] Roetten, K. and Sabates-Wheeler, R. (2011) A Child Sensitive Approach to Social Protection: Serving Practical and Strategic Needs. Proceedings of the Conference "Social Protection for Social Justice", London, 13-15 April 2011.

[26] Dercon, S. (2011) Social Protection, Efficiency and Growth. CSAE Working Paper WPS/2011-17, Oxford.

[27] Wagstaff, A. (2002) Poverty and Health Sector Inequalities. Bulletin of the World Health Organization, 80, 97-105.

[28] Wagstaff, A. (2003) Child Health on a Dollar a Day: Some Tentative Cross-Country Comparisons. Social Science \& Medicine, 57, 1529-1538. http://dx.doi.org/10.1016/S0277-9536(02)00555-5

[29] Deolalikar, A. (2004) Poverty and Child Malnutrition in Bangladesh. The World Bank, Washington DC.

[30] Navaneetham, K. and Jose, S. (2005) Poverty, Malnutrition and Mortality in South Asia: A Review of Issues and Options. Proceedings of CICRED Seminar on Mortality as Both a Determinant and a Consequence of Poverty and Hunger, Thiruvananthapuram, 23-25 February 2005, 1-21.

[31] Svedberg, P. (2000) Poverty and Under-Nutrition: Theory, Measurement and Policy. Oxford University Press, Oxford. http://dx.doi.org/10.1093/0198292686.001.0001

[32] Svedberg, P. (2006) Declining Child Malnutrition: A Reassessment. International Journal of Epidemiology, 35, 1336- 
1346. http://dx.doi.org/10.1093/ije/dyl157

[33] Svedberg, P. (2008) Why Malnutrition in Shining India Persists. 4th Annual Conference on Economic Growth and Development, New Delhi.

[34] Fotso, J.C. (2006) Child Health Inequities in Developing Countries: Differences across Urban and Rural Areas. International Journal for Equity in Health, 5, 9. http://dx.doi.org/10.1186/1475-9276-5-9

[35] Hong, R. and Mishra, V. (2006) Effect of Wealth Inequality on Chronic Under-Nutrition in Cambodian Children. Journal of Health and Population Nutrition, 24, 89-99.

[36] Giashuddin, M.S., Kabir, M. and Hasan, M. (2005) Economic Disparity and Child Nutrition in Bangladesh. Indian Journal of Pediatrics, 72, 481-487. http://dx.doi.org/10.1007/BF02724424

[37] Larrea, C. and Freire, W. (2002) Social Inequality and Child Malnutrition in Four Andean Countries. Revista Panamericana de Salud Pública, 11, 356-364. http://dx.doi.org/10.1590/S1020-49892002000500010

[38] Poel, E., Hosseinpoor, A., Speybroeck, N., Ourti, T. and Vega, J. (2008) Socioeconomic Inequality in Malnutrition in Developing Countries. Bulletin of the World Health Organization, 86, 282-291. http://dx.doi.org/10.2471/BLT.07.044800

[39] Pongou, R., Ezzati, M. and Salomon, J.A. (2006) Household and Community Socioeconomic and Environmental Determinants of Child Nutritional Status in Cameroon. BMC Public Health, 6, 98. http://dx.doi.org/10.1186/1471-2458-6-98

[40] Smith, L.C. and Haddad, L. (1999) Explaining Child Malnutrition in Developing Countries: A Cross-Country Analysis. Food Consumption and Nutrition Division Discussion Paper 60, International Food Policy Research Institute, Washington DC.

[41] Taguri, A., Betilmal, I., Mahmud, S.M., Ahmed, A.M., Goulet, O., Galan, P. and Hercberg, S. (2008) Risk Factors for Stunting among Under-Fives in Libya. Public Health Nutrition, 12, 1141-1149. http://dx.doi.org/10.1017/S1368980008003716

[42] Zere, E. and McIntyre, D. (2003) Inequities in Under-Five Child Malnutrition in South Africa. International Journal for Equity in Health, 2, 7. http://dx.doi.org/10.1186/1475-9276-2-7

[43] Gravelle, H. (2003) Measuring Income Related Inequality in Health: Standardisation and the Partial Concentration Index. Health Economics, 12, 803-819. http://dx.doi.org/10.1002/hec.813

[44] Moradi, A. (2010) Estimation of the Inequality Concentration Index of Health Care Financing through Beta Lorenz Curve, the Case of Islamic Republic of Iran. Proceedings of the International Conference on Applied Economics, Athens, 26-28 August 2010.

[45] Wagstaff, A. (2005) The Bounds of the Concentration Index When the Variable of Interest Is Binary, with an Application to Immunization Inequality. Health Economics, 14, 429-432. http://dx.doi.org/10.1002/hec.953

[46] Census (2001) Government of India.

[47] Institute of Health Management Research (2010) Health Care in the Sundarbans (India): Challenges and Plan for a Better Future. www.futurehealthsystems.org

[48] Zaman, H. (1998) Who Benefits and to What Extent? An Evaluation of BRAC’s Micro-Credit Program. University of Sussex, Sussex.

[49] Zaman, H. (2000) Assessing the Poverty and Vulnerability Impact of Micro-Credit in Bangladesh: A Case Study of BRAC. The World Bank, Washington DC.

[50] Wagstaff, A. and van Doorslaer, E. (2000) Equity in Health Care Finance and Delivery. In: Culyer, A. and Newhouse, J.P., Eds., Handbook of Health Economics 1B, Elsevier Science, Amsterdam, 1803-1861. http://dx.doi.org/10.1016/S1574-0064(00)80047-5

[51] Bentley, M.E. (1988) The Household Management of Childhood Diarrhea in Rural North India. Social Science \& Medicine, 27, 75-85. http://dx.doi.org/10.1016/0277-9536(88)90165-7 
Scientific Research Publishing (SCIRP) is one of the largest Open Access journal publishers. It is currently publishing more than 200 open access, online, peer-reviewed journals covering a wide range of academic disciplines. SCIRP serves the worldwide academic communities and contributes to the progress and application of science with its publication.

Other selected journals from SCIRP are listed as below. Submit your manuscript to us via either submit@scirp.org or Online Submission Portal.
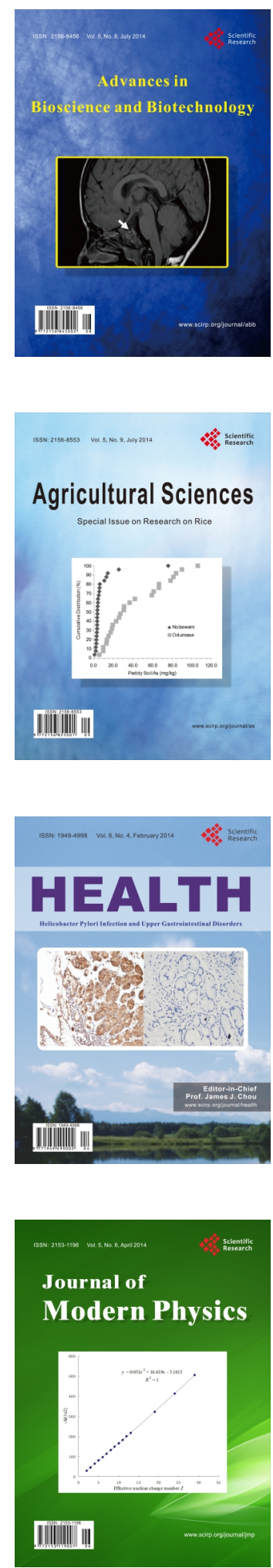
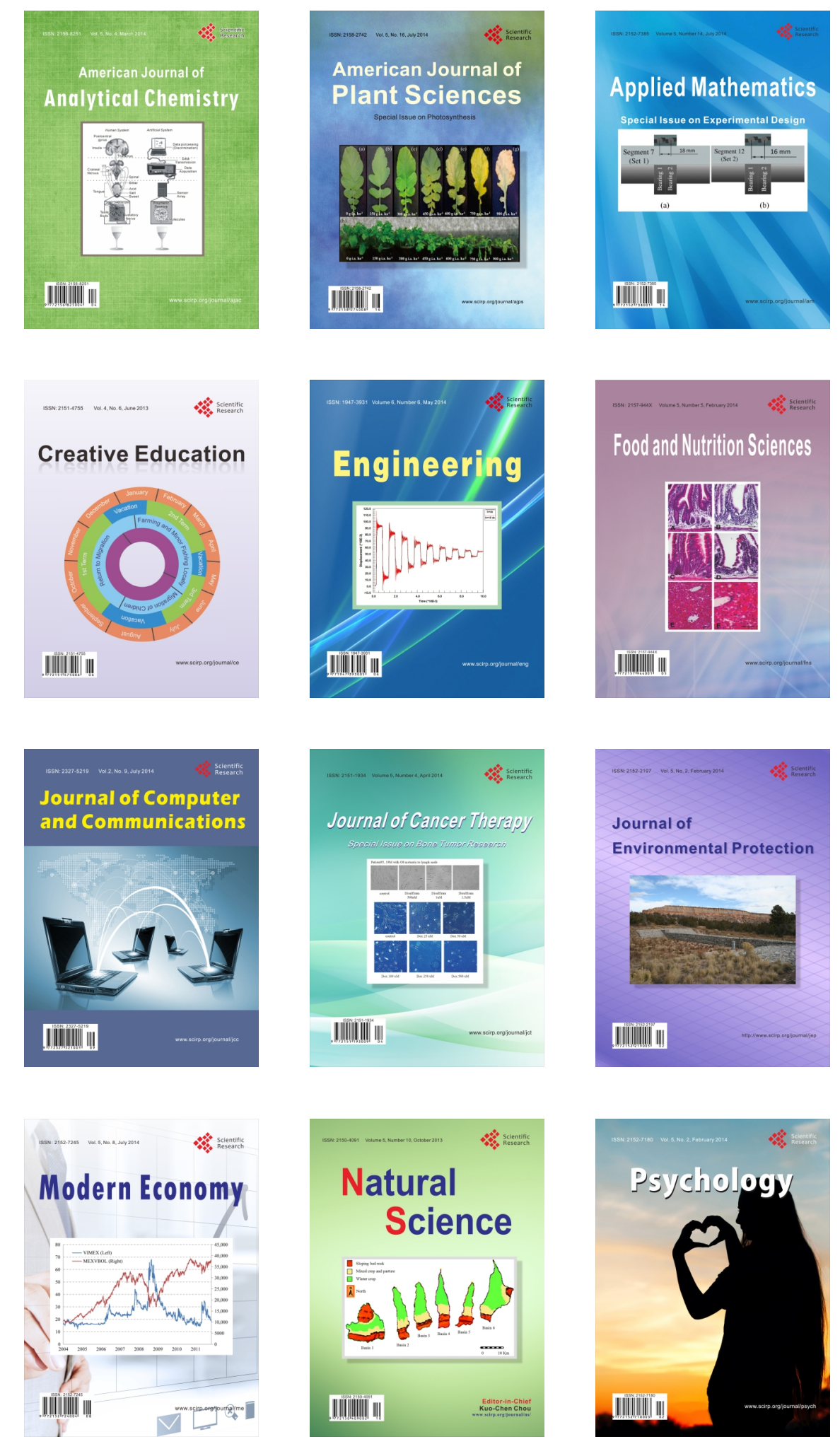